\title{
Assessment of the Level of Satisfaction and Unmet Data Needs for Specialty Drug Formulary Decisions in the United States
}

\author{
Yoonyoung Choi, MS, and Robert P. Navarro, PharmD
}

\begin{abstract}
BACKGROUND: Formulary management within a limited budget is critical, especially for specialty drugs, which are used for serious medical conditions and are very expensive. Despite attempts to summarize the pertinent evidence, it is uncertain whether data needs of formulary decision makers for specialty drugs are satisfied.
\end{abstract}

OBJECTIVE: To assess the level of satisfaction of specialty drug formulary decision makers with regards to the strength of current available data sources and unmet needs regarding clinical, economic, and unpublished evidence.

METHODS: This study targeted pharmacists and physicians involved with formulary decision making at health plans or pharmacy benefit management companies at the national, large regional, and local levels.

95 individuals were invited to participate (without compensation) in a 21-item, web-based survey (Qualtrics), which was open from June 14 to July 31,2014 . The responses were coded for descriptive and statistical analysis. Statistical analyses included the Kruskal-Wallis test, analysis of variance, and the Mann-Whitney-Wilcoxon test.

RESULTS: Of 95 pharmacists or physicians, 40 respondents initiated the survey, and 33 respondents completed the survey (response rate $=34.7 \%$ ). Drug formulary decision makers infrequently rated data evidence strength (17.1\% "always"). Clinical data evidence strength was rated highest with published randomized controlled trials (RCTs; mean [SD] = 4.06 [0.87] of 5.0), while participant organizations' internal data were rated highest for economic data evidence strength (mean $[S D]=3.91$ [1.07] of 5.0). Decision makers rated the highest unmet need as more data generated from headto-head RCTs (mean [SD] = 2.94 [0.25] of 3.0) and cost-effectiveness analyses (mean $[S D]=2.53$ [0.67] of 3.0). The participants believed manufacturers might be in the best position to satisfy their desire for head-tohead RCTs (mean $[S D]=4.31$ [1.09] of 5.0).

CONCLUSIONS: Despite a variety of data sources, drug formulary decision makers continue to rely on published RCTs or internal economic analyses as having the strongest evidence strength. The study respondents believed that pharmaceutical manufacturers would be best able to satisfy the greatest clinical data unmet need, that is, head-to-head RCTs in specialty drug formulary decisions.

J Manag Care Spec Pharm. 2016;22(4):368-75

Copyright $\odot 2016$, Academy of Managed Care Pharmacy. All rights reserved.

\section{What is already known about this subject}

Drug formularies are common in any health care organization that manages the selection and use of pharmaceuticals.

Health care professionals involved in drug formulary decisions have historically relied primarily on pharmaceutical manufacturer-sponsored registration randomized controlled trials (RCTs).
Today, health care professionals conducting drug data analyses and comparisons have access to a variety of sources of clinical and economic evidence.

\section{What this study adds}

Although clinical and economic evidence are increasingly available from a variety of data sources, drug formulary decision makers continue to rely on published RCTs as having the strongest clinical evidence strength.

Regarding economic evidence strength, drug formulary decision makers rely mostly on their own organizational internal economic analyses, perhaps because they best know their business objectives and net cost of drugs.

Pharmaceutical manufacturers appear to be in an important position to help satisfy unmet clinical data needs by including more head-to-head registration RCTs in submissions to the FDA.

$\mathrm{D}$ rug formularies for hospitals, long-term care facilities, and home care settings list available drugs and represent the compiled clinical judgment of health care providers. ${ }^{1}$ Managed care organizations have applied a similar critical drug assessment process and have adopted drug formularies as lists of covered medications to meet the specific needs of their program memberships. ${ }^{1}$ Formulary management within a limited budget is critical, especially for specialty drugs, which are used for serious medical conditions and are expensive and have a 6 times higher annual cost increase than nonspecialty drugs. ${ }^{2}$ Despite attempts to summarize the pertinent evidence for formulary management, ${ }^{3}$ it is uncertain whether data needs of formulary decision makers for specialty drugs are satisfied.

Perception of U.S. payers on data available for their decision making has drawn research attention. ${ }^{4-6}$ For many years, the only clinical data available for the review of new drugs have been from randomized controlled trials (RCTs), which are often placebo controlled and are sponsored by pharmaceutical manufacturers for drug approvals. The growing availability of comparative effectiveness research (CER) does not fully satisfy formulary decision makers because of the paucity of relevant head-to-head trials, economic data, and low reliability of manufacturer-sponsored studies. ${ }^{4}$ Pragmatic clinical trials (PCTs) have been proposed to facilitate postregulatory decisions, but RCTs are required for drug registration trials and are more 
prevalent than PCTs in the published literature. ${ }^{7}$ The value of PCTs has been acknowledged for the general population and active drug comparisons, but they do not replace RCTs or a payer's internal data analyses. ${ }^{5}$ In addition, the perception of payers regarding different types and sources of evidence has been quantified. Results indicate that RCTs and meta-analyses/ systematic reviews are valued highest. However, our investigation was limited to pharmaceutical technology assessment. ${ }^{6}$

In addition to RCTS, PCTs, and CER, ${ }^{4-6}$ clinical data and other information are available from the published literature, pharmaceutical manufacturers, third-party drug evaluation entities, the Academy of Managed Care Pharmacy (AMCP) eDossier System, ${ }^{8}$ international drug registry agencies, and other internal databases. The Food and Drug Administration Modernization Act of 1997 section 114 also provides an opportunity for pharmaceutical manufacturers to expand economic information provided to payers. ${ }^{9}$ Thus, formulary decision makers have access to several sources of data. The evaluation of these data and decision processes vary by organization, which may result in different drug coverage decisions for the same drug among various health plan and pharmacy benefit management company (PBM) formularies. This variety suggests that decision makers independently make value assessments on the strength of available data and sources.

The purpose of this study was to assess the level of satisfaction of specialty drug formulary decision makers with regards to the strength of current available data sources and unmet needs regarding clinical, economic, and unpublished evidence.

\section{Methods}

This study targeted pharmacists and physicians involved with drug evaluations and formulary decisions for specialty drugs. In this study, the term "specialty drugs" was defined as costly drugs requiring special handling and administration and was limited to brand-name drugs. Target organizations were national, multistate, large regional and local health plans; health insurers; and PBMs with employer-based commercial, Medicare Part $\mathrm{D}$, and/or Medicaid programs. Managed care organizations were identified based on 5 major sources: Utilization Review Accreditation Commission (URAC), National Committee for Quality Assurance (NCQA), Pharmacy Benefit Management Institute (PBMI), America's Health Insurance Plans (AHIP), and National Association of Insurance Commissioners (NAIC). ${ }^{10-14}$ An initial list of 54 health plans and 27 PBMs was further reviewed by one of the authors, and a final list included individuals from 89 health plans and 6 PBMs, who were involved in drug formulary management. Ninety-five individuals were invited by an e-mail to participate in an online Qualtrics survey developed by the authors of this study. This online survey encrypted survey participants and archived responses. Thus, survey responses were evaluated anonymously.
On February 24-27, 2014, a pretest to determine content validity of the survey was completed by 3 reviewers involved with formulary management. A finalized survey included the feedback obtained from this pretest. A 21-item, web-based anonymous survey was open from June 14 to July 31, 2014. After the first 2 weeks, participants received a reminder e-mail asking for survey participation if they had not yet completed the survey. The questionnaire had the following 6 major parts (see Appendix, available in online article):

1. Characteristics of survey participants (questions 1-5).

2. Strength evaluation of clinical, economic, and unpublished evidence (questions 6-8)

3. Use of evidence-rating system (questions 9-10).

4. Working practice under Food and Drug Administration Modernization Act (FDAMA) (questions 11-13).

5. Satisfaction level and unmet needs of clinical and economic data (questions 14-17).

6. Expectations of pharmaceutical companies (questions 18-21).

A 5-Likert scale was used for rating the levels of strength, frequency, and agreement. A 3-Likert scale was used for scoring usefulness and needs.

\section{Data Analysis}

The responses from each question were coded for descriptive and statistical analyses. Questions with rating scales were statistically analyzed, and responses in open text fields were collected. Statistical analyses included analysis of variance (ANOVA) and the Kruskal-Wallis test to examine differences among participants' responses. Once the 1-way ANOVA indicated the statistical significance, Kruskal-Wallis tests were also conducted, which assumed nonsymmetrical distribution and produced a higher power than the 1-way ANOVA. ${ }^{15}$ Thus, post hoc comparisons for the Kruskal-Wallis tests were provided when identifying the pairs with significant difference The SAS macro in SAS 9.4 (SAS Institute, Cary, NC) was used for this post hoc comparison. ${ }^{16}$ ANOVA and Kruskal-Wallis tests generated the mean, standard deviation [SD], median, and interquartile range. The Mann-Whitney-Wilcoxon test was used to compare the reliance level for the 2 different liaison positions with manufacturers (see Appendix, question 19). All statistical tests were conducted at a significance level of $P<0.05$ for a 2-sided test.

This research was approved by the Institutional Review Board at the University of Florida.

\section{Results}

\section{Characteristics of Survey Participants}

An online survey link was e-mailed to 95 pharmacists or physicians, who were involved with specialty drug formulary decision making. Overall, the response rate was 34.7\% (33 of 95), and approximately one third of the total responses were gener- 


\begin{tabular}{|c|c|c|c|}
\hline \multicolumn{4}{|c|}{ TABLE 1 Characteristics of Survey Participants } \\
\hline Variable & Measure & Number & Percentage \\
\hline \multirow{2}{*}{$\begin{array}{l}\text { Organization } \\
\text { type }\end{array}$} & Health plan & 31 & 77.5 \\
\hline & Pharmacy benefit manager & 9 & 22.5 \\
\hline \multirow{2}{*}{$\begin{array}{l}\text { Current } \\
\text { position }\end{array}$} & Pharmacist & 34 & 85.0 \\
\hline & Physician & 6 & 15.0 \\
\hline \multirow{3}{*}{$\begin{array}{l}\text { Level of } \\
\text { organization }\end{array}$} & National & 17 & 42.5 \\
\hline & Multistate or large regional & 14 & 35.0 \\
\hline & Local & 9 & 22.5 \\
\hline \multirow{5}{*}{$\begin{array}{l}\text { Primary lines } \\
\text { of business }\end{array}$} & Commercial or employer-based & 36 & 90.0 \\
\hline & Medicaid & 19 & 47.5 \\
\hline & Medicare & 23 & 57.5 \\
\hline & Exchange & 17 & 42.5 \\
\hline & Other & 3 & 7.5 \\
\hline \multirow{3}{*}{$\begin{array}{l}\text { Number of } \\
\text { covered lives }^{\mathrm{b}}\end{array}$} & $<500,000$ & 12 & 30.0 \\
\hline & $500,000-4,999,999$ & 16 & 40.0 \\
\hline & $>5,000,000$ & 12 & 30.0 \\
\hline \multicolumn{4}{|c|}{$\begin{array}{l}\text { aThe answers from primary lines of business were not summed to 40, since respon- } \\
\text { dents were allowed to tick all that applied. } \\
\text { The number of covered lives had a subset: }<25,000(n=3), 250,000-499,999 \\
(n=9), 500,000-999,999(n=4), 1,000,000-2,999,999(n=8), 3,000,000- \\
4,999,999(n=4), 5,000,000-9,999,999(n=4), 10,000,000-20,000,000(n=3) \text {, } \\
\text { and }>20,000,000(n=5) \text {. }\end{array}$} \\
\hline
\end{tabular}

ated after sending a reminder. Forty-three respondents opened the survey (view rate $=43$ of 95, 45.3\%); 40 respondents initiated the survey (participation rate $=40$ of $43,93.0 \%$ ); and 33 participants completed (completion rate $=33$ of $40,82.5 \%$ ) the survey. ${ }^{17}$ The majority of survey participants were pharmacists $(85.0 \%, \mathrm{n}=34)$; most participants worked at health plans $(77.5 \%, \mathrm{n}=31)$; and the primary line of business was commercial or employer-based $(90.0 \%, \mathrm{n}=36)$. Most respondents were employed by multistate or national organizations $(77.5 \%$, $\mathrm{n}=31$ ). Most organizations covered a minimum of 0.5 million lives $(70.0 \%, \mathrm{n}=28$; Table 1$)$. The summed covered lives by all surveyed organizations, based on the participants' reported membership range in survey question 5 (see Appendix), were estimated to be 170 million at minimum, representing more than half of the U.S. population.

\section{Frequency of Rating the Evidence Strength for Specialty Drug Evaluations}

When asked how frequently an evidence strength grading system was applied, the most common answer was "never" (45.7\%, $\mathrm{n}=16$ ), followed by "sometimes, when data are controversial" (28.6\%, $\mathrm{n}=10)$; "always" (17.1\%, $\mathrm{n}=6)$; "sometimes, for certain drug types" $(5.7 \%, \mathrm{n}=2)$; and "sometimes, when quantity of data is insufficient" $(2.9 \%, \mathrm{n}=1)$. Two respondents indicated in the open text field that ratings were used for a "very high cost, new entry in crowded therapeutic category" and "depends on the study and transparency." Respondents who answered "always or sometimes," were asked about the frequency of specific evidence-strength rating systems in a 5-Likert scale ("never use," "rare use," "occasional use," "frequent use," and "always use"). The frequency did not vary across the following rating systems: (a) Grades of Recommendation Assessment, Development and Evaluation (GRADE) system (mean $[S D]=3.24$ [1.15]); (b) assessment of the evidence for health care decision makers offered by the AMCP/ISPOR CER Collaborative $^{18}$ (mean $[S D]=3.06[0.77]$ ); and (c) Delfini evidence tool kit (mean $[S D]=2.31[1.11]$ ). Other than the given rating systems, a respondent answered "PBM analysis" with "occasional use" in an open text field.

\section{Perceived Strength of Clinical, Economic, and Unpublished Evidence}

Clinical Data Sources. The perceived clinical evidence strength of 10 different sources or types was evaluated using a 5-Likert scale ("very weak," "weak," "moderate," "strong," and "very strong"). "Published RCTs (randomized controlled trials)" were the strongest (mean $[\mathrm{SD}]=4.06[0.87]$ ) and "unpublished research presented at scientific meetings" were the weakest sources (mean $[S D]=2.24[0.75]$; Table 2). The KruskalWallis test showed that perceived strength is significantly different across data sources $(P<0.001)$. According to a post hoc analysis, "government sources" (e.g., the U.S. Food and Drug Administration [FDA], Centers for Disease Control and Prevention, and National Institutes of Health ) and "external clinical reviews" (e.g., Cochrane, Hayes, and the National Institute for Health and Care Excellence) were also recognized as relatively strong sources, showing no difference from published RCTs (Figure 1).

Economic Data Sources. The strength of economic data was also perceived differently among 10 different sources or types (Kruskal-Wallis test, $P<0.001$ ). "Internal financial analysis of own data" was the strongest (mean $[\mathrm{SD}]=3.91$ [1.07]), while "unpublished information provided directly by the manufacturer" (interpreted as "data on file") was the weakest data source (mean $[S D]=1.87$ [0.94]; Table 2). Based on a post hoc analysis, "external economic reviews," "published cost-effectiveness analysis," and "PBM economic reviews and recommendations" were also perceived to be relatively strong evidence (Figure 1).

Unpublished Data Sources. Unpublished data sources did not show any significant differences in perceived strength (Kruskal-Wallis test, $P=0.512$ ): "pharmaceutical company" (mean $[S D]=2.52$ [1.00]), "podium presentations" (mean $[\mathrm{SD}]=2.55[0.90]$ ), or "posters or abstracts" (mean $[\mathrm{SD}]=2.79$ [0.77]) at medical/pharmacy professional meetings and "information from colleagues from other health plans or PBMs" (mean $[S D]=2.55[0.87]$ ). Additionally, in a descriptive field, 1 respondent noted "national expert panels such as American Association for the Study of Liver Diseases treatment guidelines" with "very strong" strength. 
Clinical Evidence

KW $P<0.001$

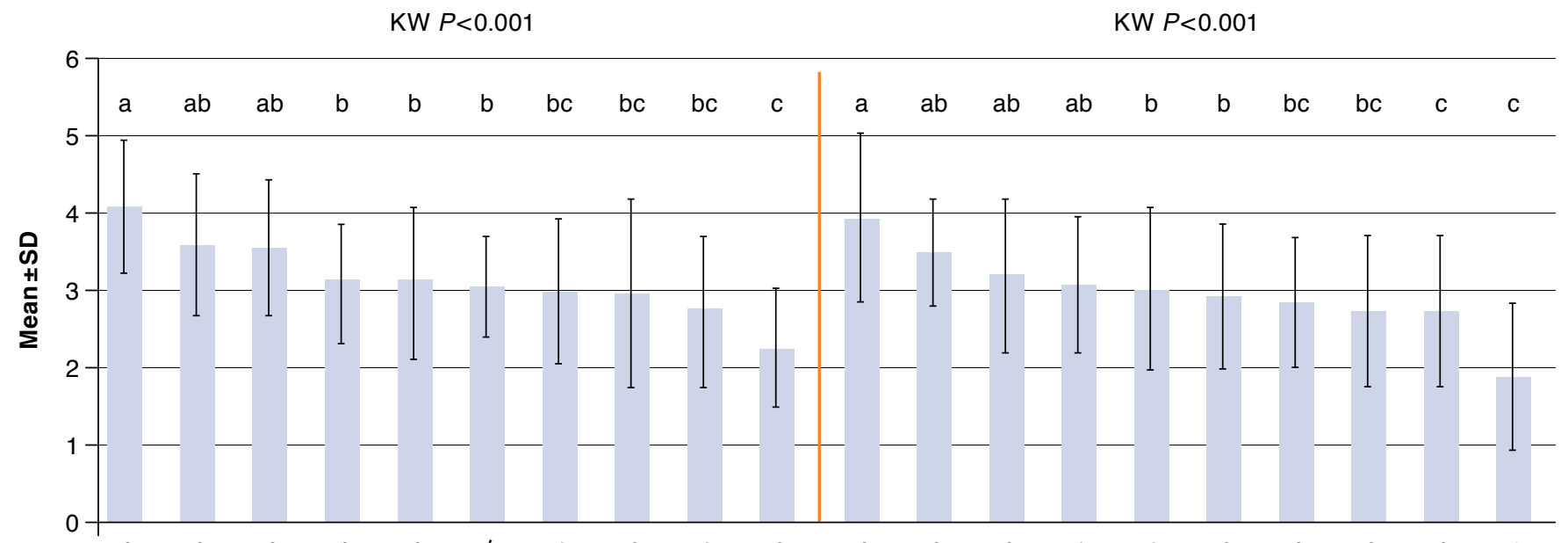

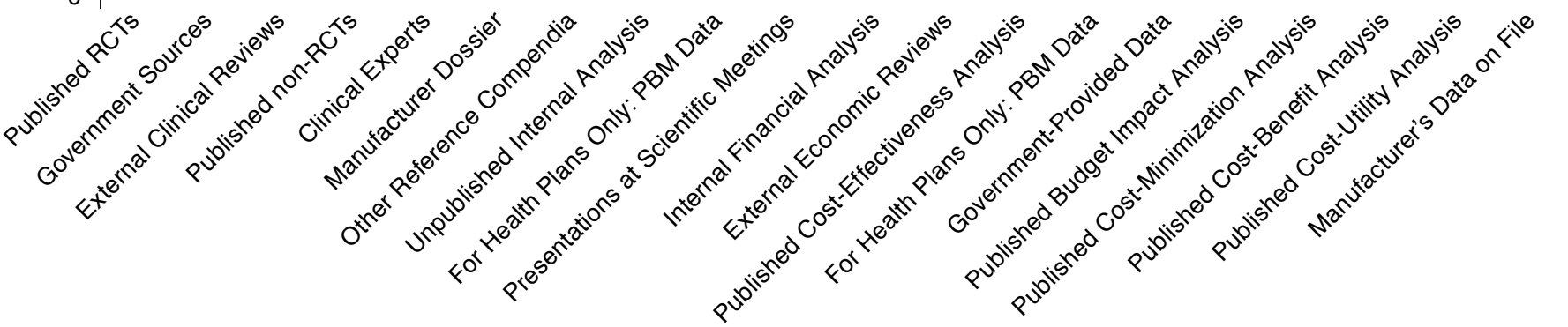

Note: Different alpha letters (alone or in combination) indicate significant differences between each data source (e.g., data source a was significantly different than data source $b$ and data source $c$, as well as the alpha letter pair $b c$, which does not contain letter a). However, data sources with the same letter (alone or in alpha letter pairs) were not significantly different (e.g., source a is not significantly different than source ab, which contains letter a).

$\mathrm{KW}=$ Kruskal-Wallis test; $\mathrm{PBM}=$ pharmacy benefit manager; $R C T=$ randomized controlled trial; $S D=$ standard deviation.

\section{Satisfaction Level and Unmet Needs of Clinical and Economic Evidence}

Respondents rated satisfaction level for 7 data attributes related to clinical evidence and economic evidence, using separate 10-point slider questions. The 7 attributes for clinical evidence (survey question 14, see Appendix) assessed satisfaction in terms of the amount, accessibility, internal and external validity, interventions and exposures (e.g., active comparators), statistical analysis, and interpretation of results. Clinical data satisfaction level was different by data attributes (KruskalWallis test, $P<0.001$ ), and "accessibility to data" received the most satisfied level (mean $[S D]=6.12[1.95]$ ). Post hoc analysis discriminated the least satisfactory attribute, which had relevance to "intervention/exposures" (mean $[S D]=3.32[2.46]$ ). For economic evidence (survey questions 16), the satisfaction level was not significantly different among attributes (KruskalWallis test, $P=0.348$; Figure 2).

When participants were asked for recommendations on improving clinical data available for formulary decision making, they rated "head-to-head RCTs" as the highest need (mean $[\mathrm{SD}]=2.94[0.25]$, using a 3-Likert scale ("low need," "medium need," and "high need"). "Observational studies" (mean $[\mathrm{SD}]=2.16[0.58]$ ) and "meta-analysis" (mean $[\mathrm{SD}]=1.75$ [0.67]) were rated as lower need (Kruskal-Wallis test, $P<0.001$ ). A single respondent answered "Better safety surveillance" in the open text field, with a rating of "medium need." Among economic evidence sources, more data from "CEA" (costeffectiveness analysis, mean $[\mathrm{SD}]=2.53[0.67]$ ) or "CBA" (costbenefit analysis, mean $[\mathrm{SD}]=2.18$ [0.73]) were in high demand, while "CUA" (cost-utility analysis, mean $[\mathrm{SD}]=1.79$ [0.99]) or "cost-analysis" (mean $[\mathrm{SD}]=1.94[0.79]$ ) were rated as a relatively lower need (Kruskal-Wallis test, $P=0.002$ ).

\section{Food and Drug Administration Modernization Act of 1997 Section 114}

More than half of the survey participants were aware of the Food and Drug Administration Modernization Act of 1997 (FDAMA; $55.9 \%, \mathrm{n}=19$ ). The proportion of respondents who 
TABLE 2 Perceived Strength of Clinical, Economic, and Unpublished Evidence

\begin{tabular}{|c|c|c|c|c|c|}
\hline Data Sources & Number & Mean & SD & Median & IQR \\
\hline \multicolumn{6}{|l|}{ Clinical } \\
\hline Published RCT studies & 35 & 4.06 & 0.87 & 4 & 2.00 \\
\hline Government sources (e.g., FDA, CDC) & 32 & 3.59 & 0.91 & 4 & 1.00 \\
\hline External clinical reviews (e.g., Cochrane, Hayes, NICE) & 32 & 3.53 & 0.88 & 4 & 1.00 \\
\hline Published non-RCT studies (e.g., meta-analyses, CER) & 33 & 3.09 & 0.77 & 3 & 1.00 \\
\hline Clinical expert colleagues & 34 & 3.09 & 0.97 & 3 & 1.75 \\
\hline Manufacturer dossier (e.g., AMCP Format) & 35 & 3.03 & 0.66 & 3 & 0.00 \\
\hline Other reference compendia (e.g., Micromedex, AHFS) & 32 & 2.97 & 0.93 & 3 & 0.25 \\
\hline Unpublished internal analysis & 31 & 2.94 & 1.21 & 3 & 2.00 \\
\hline $\begin{array}{l}\text { For health plans only: } \\
\text { PBM formulary reviews and recommendations }\end{array}$ & 22 & 2.73 & 0.98 & 3 & 1.00 \\
\hline Unpublished research presented at scientific meetings & 33 & 2.24 & 0.75 & 2 & 1.00 \\
\hline \multicolumn{6}{|l|}{ Economic } \\
\hline Internal financial analysis of own data & 35 & 3.91 & 1.07 & 4 & 2.00 \\
\hline External economic reviews & 31 & 3.48 & 0.68 & 4 & 1.00 \\
\hline Published cost-effectiveness analysis & 34 & 3.18 & 0.97 & 3 & 1.00 \\
\hline $\begin{array}{l}\text { For health plans only: } \\
\text { PBM economic reviews and recommendations }\end{array}$ & 20 & 3.05 & 0.89 & 3 & 0.25 \\
\hline Government-provided data & 31 & 3.00 & 1.06 & 3 & 2.00 \\
\hline Published budget impact analysis & 28 & 2.89 & 0.92 & 3 & 2.00 \\
\hline Published cost-minimization analysis & 32 & 2.84 & 0.85 & 3 & 1.00 \\
\hline Published cost-benefit analysis & 32 & 2.72 & 0.96 & 3 & 1.00 \\
\hline Published cost-utility analysis & 31 & 2.71 & 0.97 & 3 & 1.00 \\
\hline Unpublished information provided by the manufacturer & 30 & 1.87 & 0.94 & 2 & 2.00 \\
\hline \multicolumn{6}{|l|}{ Unpublished } \\
\hline Pharmaceutical company & 33 & 2.52 & 1.00 & 3 & 1.00 \\
\hline Professional meeting, podium presentations & 33 & 2.55 & 0.90 & 3 & 1.00 \\
\hline Professional meeting, posters or abstracts & 34 & 2.79 & 0.77 & 3 & 1.00 \\
\hline Information from colleagues from other health plans or PBMs & 33 & 2.55 & 0.87 & 3 & 1.00 \\
\hline
\end{tabular}

had received data from manufacturers specifically under a FDAMA disclosure varied by data type: on-label published data about the clinical and economic value of drugs (72.2\%, $\mathrm{n}=13)$, on-label but unpublished data $(61.1 \%, \mathrm{n}=11)$, and offlabel data $(55.6 \%, n=10)$. These respondents answered questions about the usefulness of these data types using a 3-Likert scale ("low usefulness," "medium usefulness," and "high usefulness"). The usefulness of these data types was not rated very high: "published on-label data" (mean $[S D]=1.92$ [049]), "unpublished on-label data" (mean [SD = 0.45 [0.52]), and "offlabel data" (mean $[S D]=1.50[0.53]$ ).

Pharmaceutical Manufacturers' Potential to Fill Unmet Needs Survey participants were also asked how pharmaceutical manufacturers could satisfy the unmet data needs for brand specialty drugs. The agreement level was significantly different by data type (Kruskal-Wallis test, $P<0.001$ ). According to post hoc comparisons, "head-to-head RCTs" received the highest agreement level (mean $[S D]=4.31[1.09]$ ) as a way that pharmaceutical companies could satisfy knowledge gaps. Data sources with lower agreement levels included "observational studies" (mean [SD] = 3.28 [0.99]), "cost-effective analysis" (mean $[S D]=3.09[1.12]$ ), "cost-utility analysis" (mean $[S D]=2.91$ [1.15]), and "meta-analysis" (mean [SD] = 2.81 [0.82]).

More specifically, participants indicated circumstances in which formulary decision makers valued information from pharmaceutical company contacts such as medical science liaisons (MSLs) or health outcomes liaisons (HOLs). Such clinical and/or economic interactions were most valued for drugs with unique and novel mechanisms of action, followed by noncancer unique orphan drugs, cancer drugs, and drugs with data only available from manufacturers. Regarding comparison between HOLs and MSLs, MSLs were perceived more reliable sources (mean $[\mathrm{SD}]=3.50[0.80]$ ) to attain balanced, accurate, and compelling data than HOLs (mean $[\mathrm{SD}]=2.81$ [0.80]; MannWhitney-Wilcoxon test, $P=0.002$ ) in a 5-Likert scale ("never," "unlikely," "sometimes," "frequently," and "almost always"). 


\section{FIGURE 2 Satisfaction Level of Clinical and Economic Evidence (Post Hoc Comparisons)}

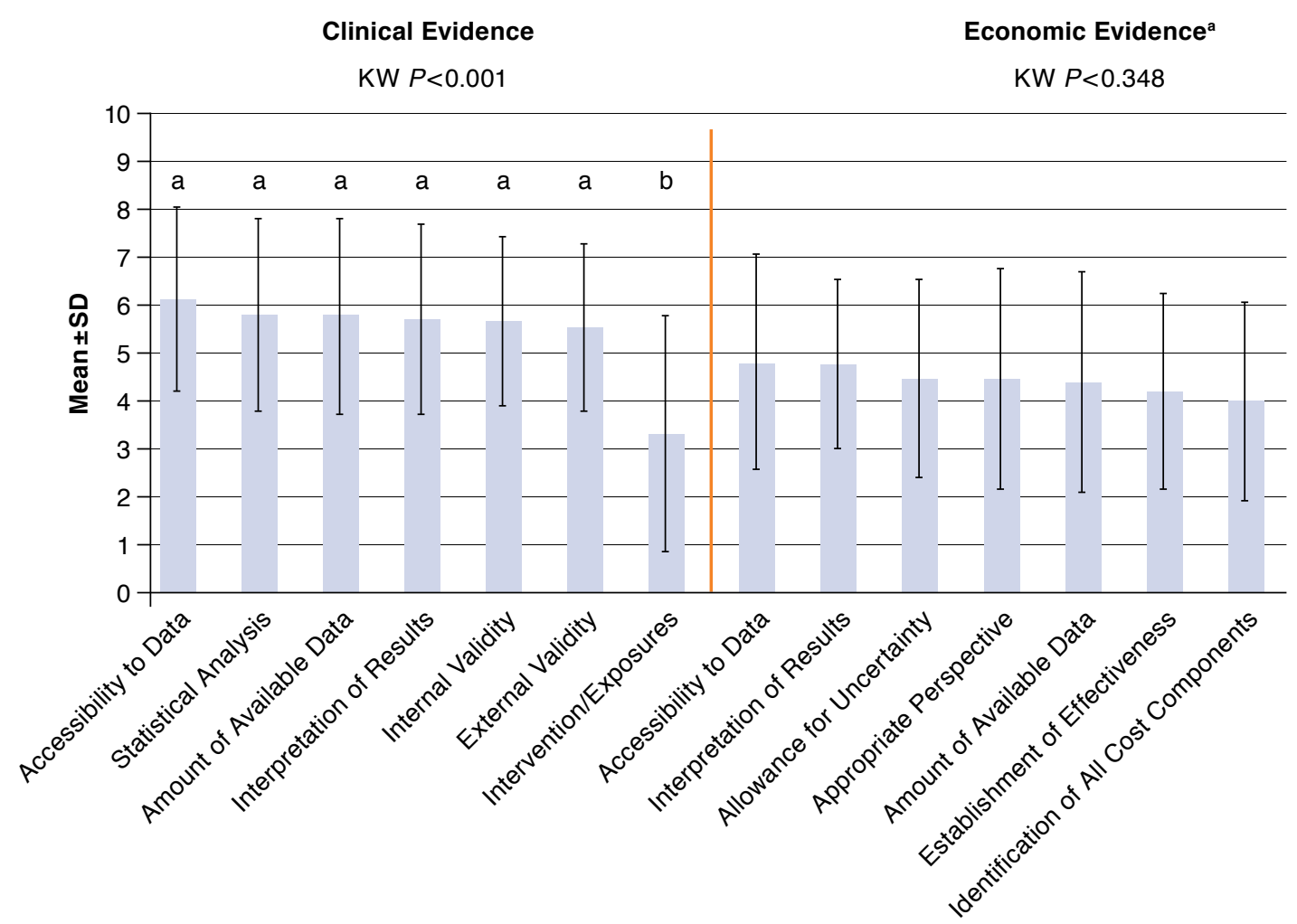

Note: Different alpha letters indicate significant differences between each data source (e.g., data source a was significantly different than data source b). However, data sources with the same letter were not significantly different.

a For economic data, post hoc comparison was not conducted because KW indicated nonsignificance.

$K W=$ Kruskal-Wallis test $; \mathrm{SD}=$ standard deviation .

\section{Discussion}

Specialty drug management is a serious concern for U.S. payers because of high patient cost and increasing availability of specialty drugs, ${ }^{19}$ which are estimated to account for half of the total drug spend by $2018 .^{20}$ This study described how payers perceived currently available clinical and economic evidence for formulary management of specialty drugs. Published RCT data were the highest rated clinical data sources for evidence strength. This finding is consistent with a previous study focusing on pharmaceutical technology assessment. ${ }^{6}$ In fact, this rating was not surprising, since RCTs have been standard in evaluating the efficacy and safety of drugs and providing evidence for FDA approvals. ${ }^{21}$ On the opposite end of the spectrum, the perception of unpublished data as the weakest type of evidence reflects payer concerns regarding incomplete or misleading information.

When evaluating economic data sources, drug formulary decision makers relied mostly on their own organizational internal economic analyses, presumably because they best knew their business objectives and net cost of drugs. Published economic sources may be supplemental, but they alone might not be sufficient for benefit design and formulary decisions on pharmacy benefit financial exposure. Pharmaceutical manufacturers often produce budget impact models (BIMs) as part of the AMCP dossier or as stand-alone economic models to supplement payer drug analyses. Study participants rated the strength of BIMs lower than their own internal analyses but at approximately the same level as other economic methodologies (e.g., cost-benefit analyses or cost-minimization analyses). BIMs are often considered to be less reliable because they are funded by pharmaceutical manufacturers. ${ }^{22}$

Similarly, the evidence strength of manufacturers' data on file was perceived as weak because of a possible lack of data transparency. ${ }^{23}$ The lower strength of CUAs shown in this study might be because the output of CUAs is usually quality-adjusted life years (QALYs), which are not commonly used by U.S. payers at this time. However, this may change as the economic training and experience of decision makers grow, and more and more plans adopt value-based drug formularies. ${ }^{24}$ 
When asked how unmet clinical data needs may be satisfied, the highest response was head-to-head RCTs. Since pharmaceutical manufacturers have been the source of RCTs, the participants believed that manufacturers might be in the best position to provide head-to-head RCTs. One interpretation of this high rating is that pharmaceutical manufacturers should include an active comparator in the registration RCTs to be submitted for FDA approval. This is often requested by U.S. payers but, unfortunately, is an infrequent occurrence, presumably because of the high cost and risk associated with active comparator RCTs. Other data sources that were rated beneficial for formulary management included observational studies, CEAs, and CBAs. Increasing reliance on health economic or outcomes research among U.S. payers was noted in one survey. ${ }^{25}$ The funding of CER by the American Recovery and Reinvestment Act (ARRA) and the Patient Protection and Affordable Care Act (PPACA) might generate more comparative data, which was reported as an unmet need by study participants. Accordingly, payers continue to rely on published RCT data to determine efficacy but would like to have at their disposal other data that represent real-world effectiveness.

The systematic grading of evidence strength also varied considerably. For example, a minority of participants always rated data, while one-third selectively rated data. We hypothesized that inconsistent application of evidence strength grading systems may be a result of (a) lack of familiarity with evidence comparison or the use of rating systems; (b) a belief that available data strength is adequate, and rating is unnecessary; or (c) confidence in the ability to rate data without the use of an external rating system.

In our study, representatives of pharmaceutical manufacturers rarely mentioned FDAMA Section 114 when they presented scientific data. Other research has also reported that only a few manufacturers are under the FDAMA (unpublished data obtained in other research projects for the pharmaceutical industry by Robert P. Navarro from 2013 to 2015). Section 114 of the FDAMA states that data delivered to a formulary committee shall "not be false or misleading and is based on competent and reliable scientific evidence." Although Section 114 of the FDAMA has been in existence for almost 20 years, presenting data with FDAMA disclosure is rarely exercised and thus far has been considered of limited value. The reluctance of manufacturers to disclose Section 114 of the FDAMA might be due to the vague and inscrutable nature of the FDAMA itself. ${ }^{26}$ Nevertheless, drug formulary decision makers indicated their continuing reliance upon scientific information provided by pharmaceutical manufacturers' medical science liaisons (MSLs) and health economic liaisons (HOLs), especially for novel specialty drugs and orphan drugs. Despite noted need, payers considered information delivered from MSLs or HOLs as modestly reliable.

\section{Limitations}

Our study includes concerns for generalizability as well as internal validity. First, we were not able to establish reliability for our questionnaire. Internal consistency is often determined to show how well a set of questions is correlated. ${ }^{27}$ The most common reliability estimate for internal consistency, Cronbach's Alpha was not applicable for our questionnaire, since we did not summate ratings. ${ }^{28}$ However, we were able to determine content validity, ensuring that newly developed survey items provided an adequate sample of all items that potentially assess our study aims. ${ }^{27}$ Second, with a response rate of $34.7 \%$ (33 of 95), the number of survey participants was not large. We could not assess statistical significance for some questions because of the small number of participants. However, given that the overall response rate for online surveys is known to be $33 \%,{ }^{29}$ our rate of $34.7 \%$ was not very low. Also, our target population was a limited population of health plan and PBM pharmacists and physicians known, or self-reported, to be engaged in drug analysis and formulary management. Third, although survey participants were known to participate in drug formulary decisions, we did not assess their knowledge level, which might have introduced inaccuracies. Fourth, nonresponse bias might have occured if survey respondents differed from nonrespondents in meaningful ways. To minimize nonresponsiveness, we sent a survey reminder to participants. Lastly, our survey participants may not represent all payers in United States, since a limited portion of defined U.S. payers agreed to participate in this survey. When interpreting survey results, this limited generalizability should be noted.

\section{Conclusions}

Although clinical and economic evidence are increasingly available from a variety of data sources, drug formulary decision makers continue to rely mostly on published RCTs or internal economic analyses until additional reliable sources become available. Pharmaceutical manufacturers are encouraged to generate head-to-head active comparator RCTs to satisfy the obvious unmet need in clinical evidence particularly desirable for informing specialty drug formulary decisions. Health services researchers also have an opportunity to satisfy unmet economic data needs by providing well-designed CEAs or CBAs that are applicable to drug formulary specialty drug decision makers.

Drug formulary decision makers may consider their plan benefit type, organizational business objectives, and other information when making specialty drug formulary decisions. Thus, future research designs that incorporate other factors affecting formulary decisions into contemporary means of analysis may be especially helpful as clinical outcomes-based contracts begin to influence drug value assessments. 


\section{Authors}

YOONYOUNG CHOI, MS, is a Graduate Student, and ROBERT P. NAVARRO, PharmD, is Clinical Professor, Department of Pharmaceutical Outcomes and Policy, College of Pharmacy, University of Florida, Gainesville.

AUTHOR CORRESPONDENCE: Robert P. Navarro, PharmD, Department of Pharmaceutical Outcomes and Policy, College of Pharmacy, University of Florida, P.O. Box 100496, Gainesville, FL 32610. Tel.: 352.273.5526; Fax: 352.273.6270;

E-mail: rnavarro@cop.ufl.edu.

\section{DISCLOSURES}

This study was not funded by any company or pharmaceutical manufacturer. Navarro has worked as a consultant for Biogen, Purdue Pharma, and Novartis and has offered expert testimony on behalf of AstraZeneca. The authors declare no other potential conflicts of interest.

Study design was contributed primarily by Navarro, along with Choi. Choi took the lead in data collection and interpretation, assisted by Navarro. Both authors contributed equally to manuscript writing and revision.

\section{REFERENCES}

1. Tyler LS, Cole SW, May JR, et al. ASHP guidelines on the pharmacy and therapeutics committee and the formulary system. Am J Health Syst Pharm. 2008;65(13):1272-83.

2. The Express Scripts Lab. The Express Scripts 2013 drug trend report. April 2014. Available at: http://lab.express-scripts.com/ /media/7f14884da6 ef434dbf30abd82dd7e655.ashx. Accessed January 15, 2016

3. de Lissovoy G. Weighing the evidence: trends in managed care formulary decision making. J Clin Psychiatry. 2003;64(Suppl 17):29-32.

4. Wang A, Halbert RJ, Baerwaldt T, Nordyke RJ. US payer perspectives on evidence for formulary decision making. Am J Manag Care. 2012; 18(5 Spec No. 2):SP71-76.

5. Ratner J, Mullins D, Buesching DP, Cantrell RA. Pragmatic clinical trials: U.S. payers' views on their value. Am J Manag Care. 2013;19(5):e158-65.

6. Leung MY, Halpern MT, West ND. Pharmaceutical technology assessment: perspectives from payers. J Manag Care Pharm. 2012;18(3):256-64 Available at: http://www.jmcp.org/doi/abs/10.18553/jmcp.2012.18.3.256.

7. Patsopoulos NA. A pragmatic view on pragmatic trials. Dialogues Clin Neurosci. 2011;13(2):217-24.

8. Academy of Managed Care Pharmacy (AMCP). AMCP eDossier System. October 2013. Available at: https://amcp.edossiers.com/global/default.aspx Accessed January 15, 2016.

9. Food and Drug Administration Moderization Act of 1997. Pub L No. 105-115, 11 Stat 2296. Available at: http://www.fda.gov/Regulatory Information/Legislation/SignificantAmendmentstotheFDCAct/FDAMA/ default.htm. Accessed January 15, 2016.

10. Utilization Review Accreditation Commission (URAC). URAC directory of accredited companies. Available at: https://www.urac.org/directory/ DirectorySearch.aspx. Accessed January 15, 2016.

11. National Committee for Quality Assurance (NCQA). Top 20 private health insurance plans 2013-2014. Available at: http://www. ncqa.org/ReportCards/HealthPlans/HealthInsurancePlanRankings/ PrivateHealthPlanRankings20132014.aspx. Accessed January 15, 2016.

12. Pharmacy Benefit Management Institute (PBMI). PBM directory. Available at: https://www.pbmi.com/directory/pbm/. Accessed January 30, 2016.
13. America's Health Insurance Plans (AHIP). Plans and programs. Available at: https://www.ahip.org/Our-Value/Promoting-Prevention-and-Wellness/ Health-Plan-Innovations/Plans-and-Programs.aspx. Accessed January 15, 2016.

14. Heilbrunn, E. Top health insurance companies. U.S. News and World Report Health. November 5, 2014. Available at: http://health.usnews.com/ health-news/health-insurance/articles/2013/12/16/top-health-insurancecompanies. Accessed January 30, 2016.

15. Van Hecke T. Power study of anova versus Kruskal-Wallis test. J Stat Manag Syst. 2012;15(2-3):241-47.

16. Elliott AC, Hynan LS. A SAS macro implementation of a multiple comparison post hoc test for a Kruskal-Wallis analysis. Comput Methods Programs Biomed. 2011;102(1):75-80.

17. Eysenbach G. Improving the quality of web surveys: the Checklist for Reporting Results of Internet E-Surveys (CHERRIES). J Med Internet Res. 2004;6(3):e34.

18. International Society for Pharmacoeconomics and Outcomes Research (ISPOR). Assessing the evidence for health care decision makers. Available at: https://www.healthstudyassessment.org/. Accessed January 15, 2016.

19. EMD Serono. EMD Serono Specialty Digest, 11 edition. 2015. Available at: http://www.specialtydigest.emdserono.com/Default.aspx. Accessed January 15, 2016.

20. Lotvin AM, Shrank WH, Singh SC, Falit BP, Brennan TA. Specialty medications: traditional and novel tools can address rising spending on these costly drugs. Health Aff (Millwood). 2014;33(10):1736-44.

21. The impact of randomized clinical trials on health policy and medical practice: background paper. Washington, DC: U.S. Congress, Office of Technology Assessment, OTA-BP-H-22, August 1983. Available at: https:// www.princeton.edu/ ota/disk3/1983/8310/8310.PDF. Accessed January 30, 2016.

22. van de Vooren K, Duranti S, Curto A, Garattini L. A critical systematic review of budget impact analyses on drugs in the EU countries. Appl Health Econ Health Policy. 2014;12(1):33-40.

23. Kessel M. Restoring the pharmaceutical industry's reputation. Nat Biotechnol. 2014;32(10):983-90.

24. Sullivan SD, Yeung K, Vogeler C, et al. Design, implementation, and firstyear outcomes of a value-based drug formulary. J Manag Care Spec Pharm. 2015;21(4):269-75. Available at: http://www.jmcp.org/doi/abs/10.18553/ jmcp.2015.21.4.269.

25. Brixner D, Andes SA, Chillingworth K. Evidence-based medicine included in P\&T decision making process. Paper presented at: 2011 International Society for Pharmacoeconomics and Outcomes Research 16th Annual International Meeting; May 24, 2011; Baltimore, MD. [Page 4]. Available at: http://www.ispor.org/meetings/baltimore0511/presentations/ ISPOR-AMCP-presentation-FINAL-5-10-11.pdf. Accessed January 15, 2016.

26. Perfetto EM, Burke L, Oehrlein EM, Gaballah M. FDAMA Section 114: Why the renewed interest? J Manag Care Spec Pharm. 2015;21(5):368-74. Available at: http://www.jmcp.org/doi/abs/10.18553/jmcp.2015.21.5.368.

27. Kimberlin CL, Winterstein AG. Validity and reliability of measurement instruments used in research. Am J Health Syst Pharm. 2008;65(23):2276-84.

28. Gliem JA, Gliem RR. Calculating, interpreting, and reporting Cronbach's alpha reliability coefficient for Likert-type scales. Paper presented at: 2003 Midwest Research-to-Practice Conference in Adult, Continuing, and Community Education; October 8-10, 2003; The Ohio State University, Columbus, OH. Available at: http://www.ssnpstudents.com/wp/wp-content/ uploads/2015/02/Gliem-Gliem.pdf. Accessed January 15, 2016.

29. Nulty DD. The adequacy of response rates to online and paper surveys: what can be done? Assess Eval High Educ. 2008;33(3):301-14. 


\section{APPENDIX Study Questionnaire}

1. What is your organization type?

$\square$ Health plan, insurance company (any line of business)

$\square$ Pharmacy benefit manager (PBM)

2. What best describes your current position in your organization?

$\square$ A pharmacy director

$\square$ A medical director

3. Please indicate your primary lines of business? (tick all that apply)

$\square$ Commercial or employer-based (full risk or self-insured)

$\square$ Medicaid

$\square$ Medicare

$\square$ Exchange

$\square$ Other

4. Which best describes your level of organization?

$\square$ National

$\square$ Multi-state or large regional

$\square$ Local

5. How many covered lives are associated with your organization?

$\square<250,000$

$\square$ 250,000-499,999

$\square$ 500,000-999,999

$\square 1,000,000-2,999,999$

$\square 3,000,000-4,999,999$

$\square 5,000,000-9,999,999$

$\square 10,000,000-20,000,000$

$\square>20,000,000$

6. When reflecting on recent formulary decisions in your organization for brand specialty drugs, the following data sources may have been used as part of the decision-making process. Assuming they were used, please rate the general strength of CLINICAL evidence provided by each data source. (Do not consider line or patent extension dosage forms and strengths.) If not used, please tick "not applicable."

Note: Throughout this survey, "published" refers to only peer-reviewed publications.

\begin{tabular}{|c|c|c|c|c|c|c|}
\hline & Not applicable & 1 (Very weak) & 2 (Weak) & 3 (Moderate) & 4 (Strong) & 5 (Very strong) \\
\hline Published RCT studies & $\bigcirc$ & $\bigcirc$ & 0 & $\mathrm{O}$ & 0 & $\mathrm{O}$ \\
\hline $\begin{array}{l}\text { Published non-RCT studies (e.g., } \\
\text { meta-analysis, observational studies, CER) }\end{array}$ & $\bigcirc$ & $\bigcirc$ & $\bigcirc$ & $\bigcirc$ & O & O \\
\hline $\begin{array}{l}\text { Government sources (e.g., FDA, CDC, NIH, } \\
\text { National Guideline Clearinghouse) }\end{array}$ & 0 & ○ & O & O & O & 0 \\
\hline $\begin{array}{l}\text { External clinical reviews (e.g., Cochrane, } \\
\text { Hayes, NICE) }\end{array}$ & 0 & O & 0 & $\bigcirc$ & $\bigcirc$ & O \\
\hline $\begin{array}{l}\text { Other reference compendia (e.g., Micromedex, } \\
\text { AHFS) }\end{array}$ & O & $\bigcirc$ & O & O & O & ○ \\
\hline Clinical expert colleagues & O & 0 & 0 & 0 & 0 & 0 \\
\hline $\begin{array}{l}\text { Unpublished research presented at scientific } \\
\text { meetings }\end{array}$ & $\bigcirc$ & $\bigcirc$ & $\bigcirc$ & $\bigcirc$ & $\bigcirc$ & 0 \\
\hline $\begin{array}{l}\text { For health plans only: PBM formulary } \\
\text { reviews and recommendations }\end{array}$ & 0 & 0 & 0 & 0 & 0 & 0 \\
\hline
\end{tabular}




\section{APPENDIX Study Questionnaire (continued)}

7. When reflecting on recent formulary decisions in your organization for novel brand specialty drugs, the following data sources may have been used as part of the decision-making process. Assuming they were used, please rate the general strength of ECONOMIC evidence provided by each data source. (Do not consider line or patent extension dosage forms and strengths.) If not used, please tick "not applicable."

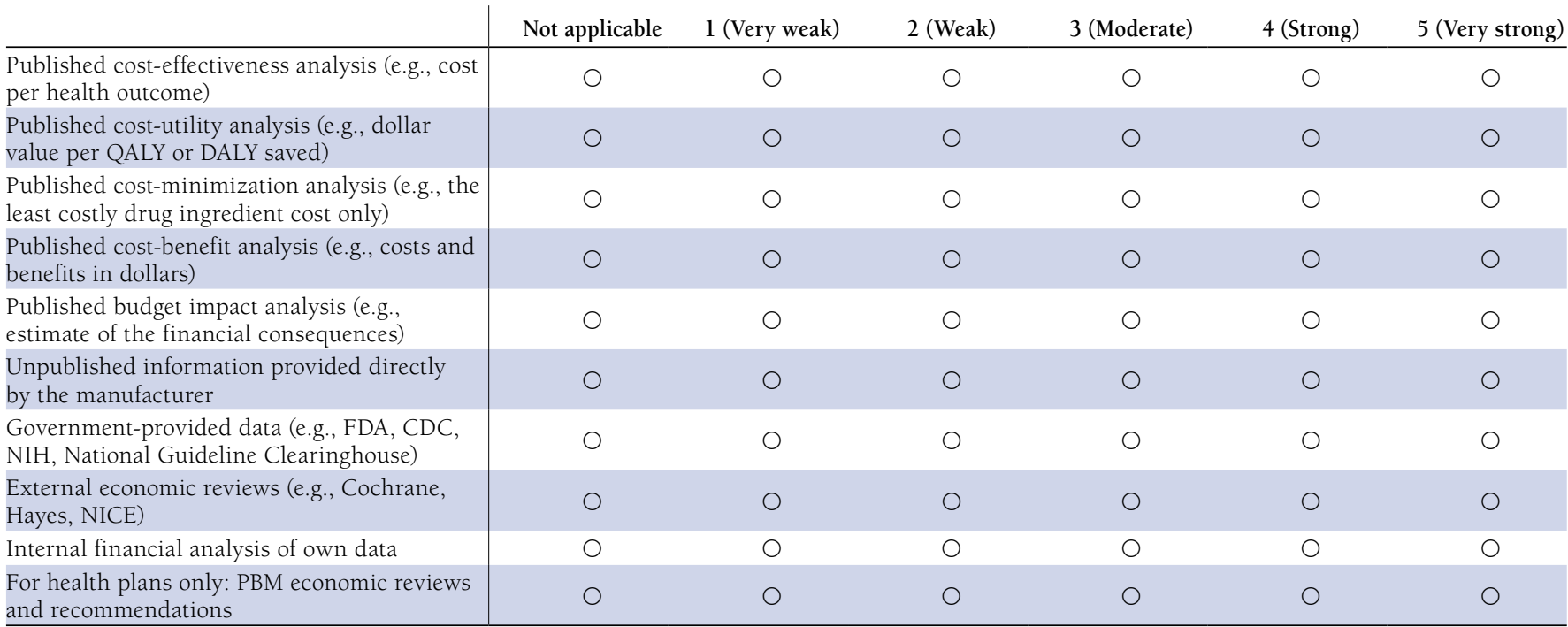

8. When reflecting on recent formulary decisions in your organization for novel brand specialty drugs, unpublished data may have been used as part of the decision-making process. Assuming they were used, please rate the general strength of clinical and/or economic evidence of UNPUBLISHED data from each source.

\begin{tabular}{|c|c|c|c|c|c|c|}
\hline & Not applicable & 1 (Very weak) & 2 (Weak) & 3 (Moderate) & 4 (Strong) & 5 (Very strong) \\
\hline $\begin{array}{l}\text { Pharmaceutical company (MSLs, Medical } \\
\text { Information, HEOR department, etc.) }\end{array}$ & O & $\bigcirc$ & $\bigcirc$ & O & ○ & ○ \\
\hline $\begin{array}{l}\text { Medical/pharmacy professional meeting(s) } \\
\text { podium presentations }\end{array}$ & 0 & 0 & 0 & O & 0 & O \\
\hline $\begin{array}{l}\text { Medical/pharmacy professional meeting(s) } \\
\text { poster or abstracts }\end{array}$ & ○ & ○ & O & O & ○ & O \\
\hline $\begin{array}{l}\text { Information from colleagues from other } \\
\text { health plans or PBMs }\end{array}$ & O & O & O & O & O & O \\
\hline
\end{tabular}

MSL= medical science liaison; HEOR = health economics and outcomes research

9. Does your organization rate the strength of evidence using a rating system (e.g., Delfini, GRADE, USPSTF, others) when considering information collected from the above-mentioned data sources when evaluating clinical and/or economic data for brand specialty drugs?

$\square$ Yes, always rate the strength of evidence.

$\square$ No, never rate the strength of evidence.

$\square$ Sometimes, for certain drug types (Please provide examples in text entry).

$\square$ Sometimes, when data are controversial.

$\square$ Sometimes, when quantity of data is insufficient.

$\square$ Other situations (Please provide examples in text entry).

10. If you answered either YES or SOMETIMES for using a rating system, please identify how frequently you used each of the rating systems below when reflecting on recent formulary decisions in your organization for novel brand specialty drugs.

\begin{tabular}{|c|c|c|c|c|c|}
\hline & 1 (Never use) & 2 (Rare use) & 3 (Occasional use) & 4 (Frequent use) & 5 (Always) \\
\hline$\overline{\text { Delfini }}$ & 0 & 0 & 0 & 0 & 0 \\
\hline $\begin{array}{l}\text { Grades of Recommendation Assessment, } \\
\text { Development and Evaluation (GRADE) } \\
\text { system }\end{array}$ & ○ & $\bigcirc$ & $\bigcirc$ & $\bigcirc$ & 0 \\
\hline AMCP/ISPOR CER Collaborative & O & $\mathrm{O}$ & O & O & O \\
\hline Others & 0 & 0 & 0 & 0 & 0 \\
\hline
\end{tabular}




\section{APPENDIX Study Questionnaire (continued)}

11. Before today, were you aware of FDAMA of 1997 Section 114 ?

FDAMA of 1997 Section 114 allows pharmaceutical manufactures to provide economic data to formulary decision makers for labeled indication

$\square$ Yes

$\square$ No

12. Please indicate if a pharmaceutical company representative told you that clinical or economic data for brand specialty drugs were (a) presented to you specifically under FDAMA section 114, and if presented, indicate (b) how USEFUL was it for formulary decision making?

\begin{tabular}{|c|c|c|c|c|}
\hline & Not presented & 1 (Low usefulness) & $\begin{array}{l}2 \text { (Medium } \\
\text { usefulness) }\end{array}$ & $\begin{array}{c}3 \text { (High } \\
\text { usefulness) }\end{array}$ \\
\hline Data on off-label clinical and economic benefits of drugs & 0 & 0 & 0 & 0 \\
\hline
\end{tabular}

13. Please provide the names of the pharmaceutical companies that have provided data for brand specialty drugs specifically under FDAMA 1997 section 114 , and the number of drugs for which they have provided such data.

\begin{tabular}{|c|c|c|c|c|}
\hline & Number o & by $\mathrm{Fl}$ & on 114 & Manufacturer \\
\hline & 0 & $1-2$ & $3-4$ & 5 or more \\
\hline 1 & 0 & 0 & 0 & 0 \\
\hline 2 & 0 & 0 & 0 & 0 \\
\hline 4 & 0 & 0 & 0 & 0 \\
\hline 5 & 0 & 0 & 0 & 0 \\
\hline
\end{tabular}

14. When thinking about the CLINICAL information for brand specialty drugs available to your organization from all the data sources used for formulary decision making, how satisfied are you with each of the following:

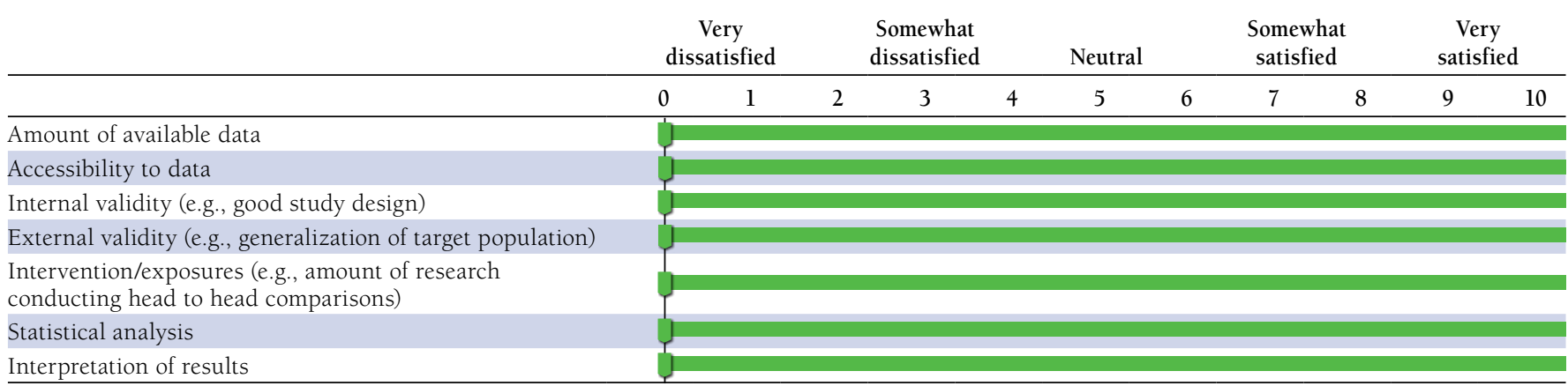

15. Please indicate whether having access to more of the following study types that offered more CLINICAL information for brand specialty drugs would be helpful in improving your formulary decision making.

\begin{tabular}{|c|c|c|c|c|}
\hline & No need & 1 (Low need) & 2 (Medium need) & 3 (High need) \\
\hline Meta-analysis & $\bigcirc$ & 0 & 0 & 0 \\
\hline Head-to-head RCTs & 0 & O & O & O \\
\hline $\begin{array}{l}\text { Observation studies (e.g., non-randomized comparative } \\
\text { effectiveness research) }\end{array}$ & $\bigcirc$ & $\bigcirc$ & ○ & O \\
\hline Others & 0 & 0 & 0 & 0 \\
\hline
\end{tabular}




\section{APPENDIX Study Questionnaire (continued)}

16. When thinking about the ECONOMIC information for brand specialty drugs available to your organization from all the data sources used for formulary decision making, how satisfied are you with each of the following:

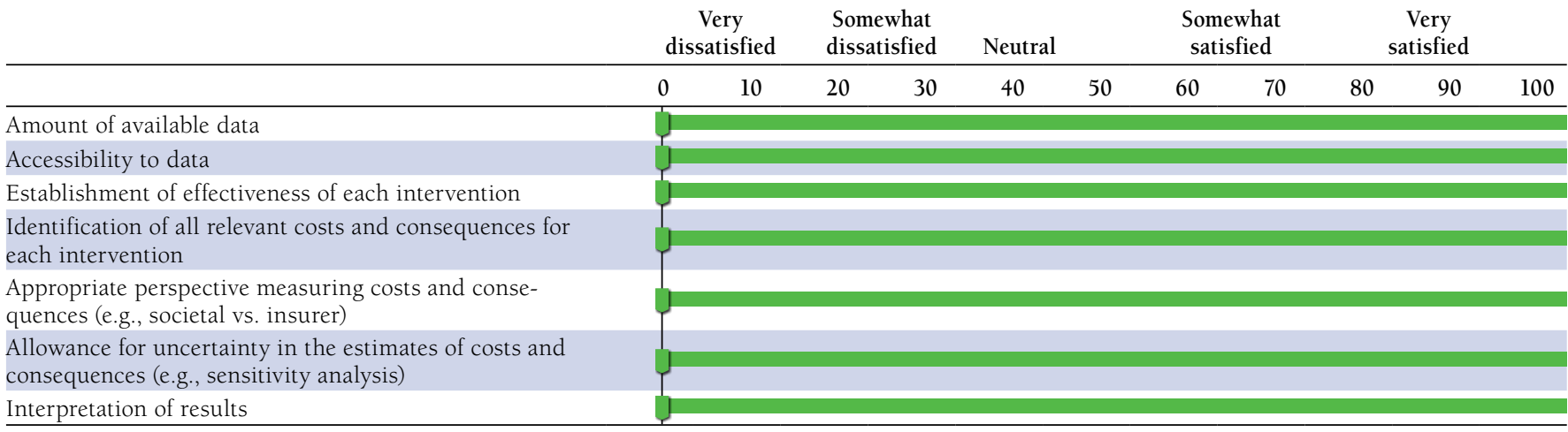

17. Please indicate whether having access to more of the following study types that offered more ECONOMIC information for brand specialty drugs would be helpful in improving your formulary decision making.

Cost analysis data (e.g., economic burden)

Cost-benefit analysis data

Cost-effectiveness analysis data

Cost-utility analysis data (QALYs)

Others

\begin{tabular}{cccc} 
No need & 1 (Low need) & 2 (Medium need) & 3 (High need) \\
\hline & 0 & 0 & 0 \\
0 & 0 & 0 & 0 \\
0 & 0 & 0 & 0 \\
0 & 0 & 0 & 0 \\
0 & 0 & 0 & 0 \\
\hline
\end{tabular}

18. Please indicate your agreement that pharmaceutical manufacturers could satisfy your unmet data needs for brand specialty drugs with the following evidence.

\begin{tabular}{|c|c|c|c|c|c|c|}
\hline & Not applicable & $\begin{array}{l}1 \text { (Strongly } \\
\text { disagree) }\end{array}$ & 2 (Disagree) & $\begin{array}{l}3 \text { (Neither } \\
\text { agree or } \\
\text { disagree) }\end{array}$ & 4 (Agree) & $\begin{array}{l}5 \text { (Strongly } \\
\text { agree) }\end{array}$ \\
\hline Meta-analysis & O & $\bigcirc$ & O & O & O & O \\
\hline $\begin{array}{l}\text { Observational studies (e.g., non-randomized } \\
\text { comparative effectiveness research) }\end{array}$ & ○ & O & $\bigcirc$ & $\bigcirc$ & O & ○ \\
\hline Cost-effectiveness analysis & 0 & O & $\bigcirc$ & $\bigcirc$ & $\bigcirc$ & 0 \\
\hline
\end{tabular}

19. What is your organization's practice for brand specialty drugs regarding Medical Science Liaisons (MSLs) and Health Outcomes Liaison (HOLs) presentations?

\begin{tabular}{|c|c|c|c|c|}
\hline & \multicolumn{4}{|c|}{ Please choose one among 4 options for each role of MSL and HOL } \\
\hline & Has never been offered & $\begin{array}{l}\text { Presentation offers are } \\
\text { generally not permitted }\end{array}$ & $\begin{array}{l}\text { Allowed only for specific } \\
\text { drugs (e.g., specialty, } \\
\text { orphan, other high cost or } \\
\text { high risk drugs, or drugs } \\
\text { with controversial data) }\end{array}$ & $\begin{array}{c}\text { Generally all presentations } \\
\text { offers are allowed }\end{array}$ \\
\hline Medical Science Liaisons (MSLs) & $\mathrm{O}$ & O & $\mathrm{O}$ & O \\
\hline Health Outcomes Liaisons (HOLs) & 0 & 0 & 0 & 0 \\
\hline
\end{tabular}




\section{APPENDIX Study Questionnaire (continued)}

20. Please RANK the value of MSL/HOL presentations as a valuable data source for brand specialty drugs. Note that 1 is most valuable and 5 is the least valuable data source. (Do not choose the same rank more than once.)

\begin{tabular}{|c|c|c|c|c|c|}
\hline & 1 & 2 & 3 & 4 & 5 \\
\hline $\begin{array}{l}\text { Any brand specialty drugs with unique or novel MOAs } \\
\text { (mechanism of actions) }\end{array}$ & $\bigcirc$ & $\bigcirc$ & $\bigcirc$ & $\bigcirc$ & O \\
\hline Cancer drugs & 0 & 0 & 0 & 0 & O \\
\hline $\begin{array}{l}\text { Drugs with unpublished data only available through } \\
\text { manufacturers }\end{array}$ & O & 0 & 0 & O & 0 \\
\hline Others: & & & & & \\
\hline
\end{tabular}

21. When encountered, are biopharmaceutical company MSLs/HOLs reliable sources to provide balanced, accurate, and compelling clinical and/or economic evidence for brand specialty drugs? Please provide your general experience regarding drug formulary decisions.

\begin{tabular}{|c|c|c|c|c|c|}
\hline & 1 (Never) & 2 (Unlikely) & 3 (Sometimes) & 4 (Frequently) & $\begin{array}{c}5 \text { (Almost } \\
\text { always) }\end{array}$ \\
\hline Economic evidence & 0 & 0 & 0 & 0 & 0 \\
\hline
\end{tabular}

